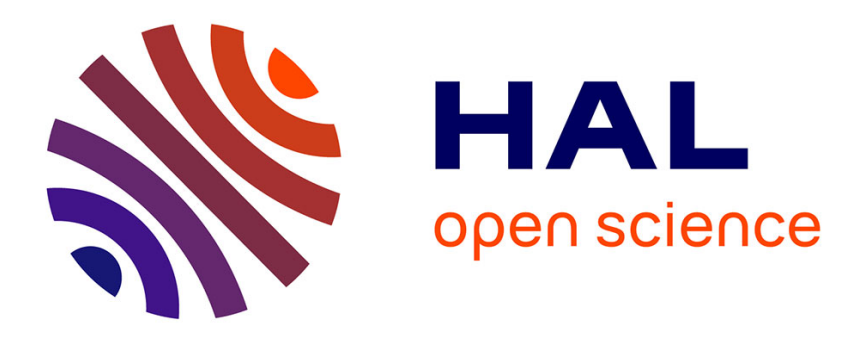

\title{
Self-association of disc-like molecules in hexadecane
}

\author{
Eric Y. Sheu, K.S. Liang, Long Y. Chiang
}

\section{To cite this version:}

Eric Y. Sheu, K.S. Liang, Long Y. Chiang. Self-association of disc-like molecules in hexadecane. Journal de Physique, 1989, 50 (10), pp.1279-1295. 10.1051/jphys:0198900500100127900 . jpa-00210994

\section{HAL Id: jpa-00210994 https://hal.science/jpa-00210994}

Submitted on 1 Jan 1989

HAL is a multi-disciplinary open access archive for the deposit and dissemination of scientific research documents, whether they are published or not. The documents may come from teaching and research institutions in France or abroad, or from public or private research centers.
L'archive ouverte pluridisciplinaire HAL, est destinée au dépôt et à la diffusion de documents scientifiques de niveau recherche, publiés ou non, émanant des établissements d'enseignement et de recherche français ou étrangers, des laboratoires publics ou privés. 
Classification

Physics Abstracts

$60.61-61.12 \mathrm{q}-61.12 \mathrm{Ex}$

\title{
Self-association of disc-like molecules in hexadecane
}

\author{
Eric Y. Sheu, K. S. Liang and Long Y. Chiang \\ Exxon research \& Engineering Co., Annandale, NJ 08801, U.S.A.
}

(Reçu le 25 août 1988, révisé et accepté le 23 janvier 1989)

\begin{abstract}
Résumé. - La self-association et les propriétés thermodynamiques de molécules discotiques dans l'hexadécane ont été étudiées à $22^{\circ} \mathrm{C}$ par difusion de neutrons aux petits angles. Les molécules étudiées sont le $2,3,6,7,10$, 11-hexapentoxytriphénylène (HET-5), le 2, 3, 6, 7, 10, 11hexanonoxytriphénylène (HET-9) et le $2,3,6,7,10,11$-hexa-undécyloxytriphénylène (HET-11) avec des concentrations comprises entre $10^{-5}$ et $10^{-3} \mathrm{M}$. Tous ces systèmes montrent une tendance très marquée à la self-association dans la gamme de concentrations étudiée. D'après diverses analyses structurales, nous trouvons que les molécules forment des agrégats qui sont de forme irrégulière à basse concentration et ont la forme de bâtonnets à plus haute concentration. La distribution de taille dérivée du premier principe d'équilibre chimique a été utilisée dans l'analyse des agrégats en forme de bâtonnets et on en a déduit les interactions intermoléculaires.
\end{abstract}

\begin{abstract}
Self-association and the related thermodynamic properties of disc-like molecules in hexadecane were investigated using small angle neutron scattering technique at $T=22{ }^{\circ} \mathrm{C}$. The disc-like molecules studied were $2,3,6,7,10$, 11-hexapentoxytriphenylene (HET-5), 2, 3, 6, 7, 10, 11-hexanonoxytriphenylene (HET-9), and 2, 3, 6, 7, 10-11-hexa-undecyloxytriphenylene (HET-11) with concentration ranging from $10^{-5} \mathrm{M}$ to $10^{-3} \mathrm{M}$. The three systems all show strong propensity of self-association at the concentration range studied. From various structural analyses we found that the molecules form irregular shape of aggregates at low concentration and become rod-like as concentration increases. A size distribution derived from the first principle of chemical equilibrium was applied to analyze the rod-like aggregate from which the intermolecular interactions was determined.
\end{abstract}

\section{Introduction.}

The aggregation of molecules in solution is a common theme of interest in many areas of science, including biology, colloid, and polymer. The most well studied examples are amphiphilic molecules which exhibit rich varieties of structures such as micelles, vesicles, and microemulsions [1-3]. In this paper, we report the results of our studies on self-association and the intermolecular interactions of disc-like molecules in oil. Specifically, we have performed small angle neutron scattering (SANS) measurements to observe the selfassociation of disc-like molecules in hexadecane, and have analyzed the results based on an equilibrium thermodynamic argument. 
The disc-like molecules have special architecture with rigid aromatic groups as the cores on which flexible aliphatic side chains are attached. In condense state, these materials exhibit interesting liquid crystalline phases, primarily as the consequence of differences in packing requirements for aromatic cores and aliphatic chains. Typically, the molecules stack into infinite columns in various two-dimension close packed lattices. Since the first report of discotic mesophase [4], extensive work has been advanced in synthesizing molecules of different architectures and elucidating their very rich mesophase behaviors [5]. So far, many studies on disc-like molecules are directed to the understanding of the interplay between the molecular architectures and the thermal ordering behaviors of the liquid crystalline mesophases $[6,7]$. The self-association of discotic molecules in solution was recently observed via electronic absorption and the optical structure of the aggregates were determined [8]. In this study, we are interested mainly in the interactions of discotic molecules in an aliphatic medium. One plausible implication of this study is to understand the formation of carbonaceous mesophase, which commonly occurs in petroleum and coal tar pitches [9, 10]. The carbonaceous mesophase is the basic precursor for making important products such as carbon fibers.

The paper is organized in the following order. In section 2 a description of the experiment is given. In section 3 the size distribution function of discotic aggregates is derived on the basis of chemical equilibrium considerations. The SANS intensity functions for different particle sizes, shapes, and polydispersities are given in section 4 . This is followed by a detailed descriptions of the experimental results and the data analysis in section 5. In section 6 we give the conclusion of this study. Appendix A gives a detail derivation of intraparticle structure factor for spheres with size distribution according to Schultz distribution function and Appendix B gives that of cylindrical particles with thermal equilibrium distribution (to be described in Sect. 3) in size.

\section{Experiment.}

The SANS experiment was conducted on Beam Line H9-B at high flux Brookhaven reactor at the Brookhaven National Laboratory. The wavelength, $\lambda$, of the incoming neutrons was tuned to be $4.5 \AA$ with $\Delta \lambda / \lambda<6 \%$ using a multilayer Ge-Mn monochromator. The detector was an area helium-3 detector of $50 \times 50 \mathrm{~cm}^{2}$ containing $128 \times 128$ pexels. By adjusting the sample to detector distance, the scattering intensity in the range of scattering vector $|\mathbf{Q}|\left(\equiv(4 \pi / \lambda) \sin (\theta / 2), \theta\right.$ is the scattering angle) from 0.018 to $0.17 \AA^{-1}$ can be reliably obtained.

The discotic samples used in this study were synthesized by a demethylation reaction followed by an alkylation of a common intermediate of $2,3,6,7,10,11$-hexamethoxytriphenylene (HET), as previously reported [11, 12]. The discotic molecules studied have alkyl chains $\mathrm{C}_{n} \mathrm{H}_{2 n+1}$ with $n=5,9$, and 11. The solvent used for SANS experiments is gold label deuterated hexadecane (Cambridge Isotope with deuterium atoms greater than $99.8 \%$ ). In mixing HET- $n$ with hexadecane, we found that samples with concentrations in the range of $10^{-4}$ to $10^{-2} \mathrm{M}$ were stable at room temperature (see Tab. I). However, it is worth noting that by cooling the sample temperature to below room temperature thread-like precipitates could be observed. This precipitation process is reversible. One can argue that the thread-like morphology of the precipitates is related to the columnar phase of pure discotics. In fact, it will be shown later that this indeed is part of the conclusions of this SANS study.

To perform SANS experiment, samples were loaded to circular quartz cells of $1 \mathrm{~mm}$ path length. The scattered intensities were collected with a statistical error less than $5 \%$. The measured intensity was corrected for the scattering from empty cell and the background and scaled for the detector sensitivity using $\mathrm{H}_{2} \mathrm{O}$ as standard sample. 
It will be shown later (see Sect. 5) that in order to differentiate different structural models in this study, it is necessary to use the absolute scattering intensity in the data analysis. Using $\mathrm{H}_{2} \mathrm{O}$ standard, the absolute intensity can be obtained by the following relation [13],

$$
\mathrm{d} \Sigma_{\mathrm{s}}^{i j} / \mathrm{d} \Omega=\frac{1-T_{\mathrm{w}}}{4 \pi T_{\mathrm{w}} t_{\mathrm{w}}} \frac{I_{\mathrm{s}}^{i j} /\left(t_{\mathrm{s}} T_{\mathrm{s}} M_{\mathrm{s}}\right)}{\left(t_{\mathrm{w}} T_{\mathrm{w}} M_{\mathrm{w}}\right)}
$$

where subscripts $\mathrm{s}$ stands for the discotic solution, w for water, and $(i, j)$ for pixel number ; $\mathrm{d} \Sigma_{\mathrm{s}} / \mathrm{d} \Omega$ is the differential cross section (i.e., absolute scattering intensity) per unit volume of the solution ; $t$ is the path length of the sample cell and $T$ is the corresponding transmission ; $I$ is the measured intensity and $M$ is the monitor counts.

\section{Equilibrium thermodynamic analysis of disk aggregation.}

In the course of analyzing SANS data from discotic solutions, we have incorporated several different kind of structural models for the aggregates. As to be seen later, the particularly successful one is the one that assumes the aggregates to be columnar shaped. This model fits the SANS data for the high concentration range $\left(\sim 3 \times 10^{-3}\right.$ to $1 \times 10^{-2} \mathrm{M}$, see Tab. I and Sect. 5). We therefore describe the columnar model in details first.

Let us consider the thermodynamic driving forces for the disk-like molecules to form aggregates in oils. In thermal equilibrium, the system contains monomers, dimers, and $N$-mer with certain number density distribution. Considering each $N$-mer as one phase and let $\mu_{1}$ and $\mu_{N}$ be the chemical potential of a monomer and a $N$-mer respectively in the solution. Following the Gibb's equilibrium thermodynamic argument, a monomer in any of the phases presented should have the same chemical potential, i.e.,

$$
N \mu_{1}=\mu_{N}
$$

By adapting the common approach used in micelle solutions $[14,15]$, the chemical potential for aggregates of aggregation number $N$ can be written as

$$
\mu_{N}=\mu_{N}^{0}+k_{\mathrm{B}} T \ln X_{N} \quad N=1,2,3, \ldots
$$

where $\mu_{N}^{0}$ is the standard part of chemical potential and $X_{N}$ is the mole fraction of $N$-mers. The second term of (Eq. (3)) represents the statistical entropy of mixing. Equation (3) is valid only for dilute systems where inter-aggregate interaction is negligible and the mixing is ideal. By substituting (Eq. (3)) into (Eq. (2)) one obtains

$$
X_{N}=\left(X_{1}\right)^{N} \exp \left[-\left(\mu_{N}^{0}-N \mu_{1}^{0}\right) / k_{\mathrm{B}} T\right] .
$$

In (Eq. (4)), $\left(X_{1}\right)^{N}$ is the probability of having $N$ molecules at the same spatial location and the Boltzmann factor, $\exp \left[-\left(\mu_{N}^{0}-N \mu_{1}^{0}\right) / k_{\mathrm{B}} T\right]$, represents the net energy gained by assembling $N$ free molecules in solution into an aggregate. For discotic molecules the origin of this energy gain comes from intermolecular interactions (see Sect. 5). Equation (4), together with the law of mass conservation, $X=\sum N X_{N}$ ( $X$ is the total monomer mole fraction in the system), are the two basic thermodynamic equations for our self-association analysis.

In order to formulate $\left(\mu_{N}^{0}-N \mu_{1}^{0}\right)$ in (Eq. (4)) we adapt the simple ladder model [16]. In this model the energy gain for adding one molecule to a $N$-mer is assumed to be constant (i.e., independent of $N$ ). Define $\delta$ to be the constant, the energy gained by the system in forming a 
dimer is then $\left(\mu_{2}^{0}-2 \mu_{1}^{0}\right)=\delta$. Similarly, when $N$ discotic molecules are stacked, the energy gained becomes $\left(\mu_{N}^{0}-N \mu_{1}^{0}\right)=(N-1) \delta$. Accordingly, (Eq. (4)) can be rewritten as

$$
\begin{aligned}
X_{N} & =\left(X_{1}\right)^{N} \exp \left[-(N-1) \delta / k_{\mathrm{B}} T\right] \\
& =\beta^{N} \exp \left(\delta / k_{\mathrm{B}} T\right)
\end{aligned}
$$

where

$$
\beta \equiv X_{1} \exp \left(-\delta / k_{\mathrm{B}} T\right) .
$$

We will call the size distribution of the aggregates given in (Eq. (6)) the thermal equilibrium distribution from what follows. By (Eqs. (6) and (7)), the total molecular concentration can be expressed in terms of $\delta$ and $\beta$,

$$
X=\sum_{N=1}^{\infty} N X_{N}=\mathrm{e}^{\delta / k_{\mathrm{B}} T} \frac{\beta}{(1-\beta)^{2}} .
$$

From (Eq. (8)) the weight average aggregation number $\bar{N}_{\mathrm{w}}$ and the number average aggregation number $\bar{N}_{N}$ can be conveniently expressed in terms of $\beta$,

$$
\bar{N}_{\mathrm{w}}=\sum_{N=1}^{\infty} N^{2} X_{N} / \sum_{N=1}^{\infty} N X_{N}=\frac{1+\beta}{1-\beta},
$$

and

$$
\bar{N}_{N}=\sum_{N=1}^{\infty} N X_{N} / \sum_{N=1}^{\infty} X_{N}=\frac{1}{1-\beta} .
$$

From these expressions, one sees that in the ladder model $\beta$ is the only parameter needed to characterize the thermal equilibrium distribution. Once the value of $\beta$ is determined, $\delta$ can be obtained using (Eq. (8)) for a given total concentration $X$. Since $\delta$ is assumed to be a constant in the ladder model, the size distribution $X_{N}$ behaves like $\beta^{N}$ (Eq. (6)). Therefore, the polydispersion becomes appreciable only if $\beta$ is close to unity. If the $\beta$ value is greater than unity, $X_{N}$ would then goes to infinite with $N$ and the system would be unstable (or precipitation would occur).

\section{SANS intensity functions.}

For a monodisperse system of particle number density $N_{\mathrm{p}}$ and particle volume $V_{\mathrm{p}}$, the scattering intensity per unit volume of the sample, $I(Q)$, can be written as [17]

$$
I(Q)=N_{\mathrm{p}} \cdot V_{p}^{2} \cdot\left(\rho_{\mathrm{p}}-\rho_{\mathrm{s}}\right)^{2} \cdot P(Q) \cdot S(Q)
$$

where $\rho_{\mathrm{p}}$ is the neutron scattering length density of the particle and $\rho_{\mathrm{s}}$ is that of the solvent. $P(Q)$ is the normalized intra-particle structure factor (i.e., $P(0)=1$ ) defined as square of the particle form factor. The functional characteristics of $P(Q)$ are determined mainly by particle shape and size. $S(Q)$ is the inter-particle structure factor, which is governed largely by the interparticle interactions. In our case, such interactions are negligible because the systems studied are in very dilute concentrations $S(Q)$ is therefore assumed to be unity. The analytical forms of $P(Q)$ are known for many types of particles $[18,19]$. For the two relevant cases in this study, particles with spherical and cylindrical shapes, their $P(Q)$ 's are listed in Appendice $\mathrm{A}$ and $\mathrm{B}$ for convenience of discusssions.

On the other hand, when particles are polydisperse in sizes, the intensity function, 
$I(Q)$, can be formulated by replacing $N_{\mathrm{p}}, V_{p}^{2}$ and $P(Q)$ in (Eq. (11)) with their weight values $\left\langle N_{\mathrm{p}}\right\rangle,\left\langle V_{\mathrm{p}}^{2}\right\rangle$, and $\langle P(Q)\rangle$ respectively,

$$
I(Q)=\left\langle N_{\mathrm{p}}\right\rangle \cdot\left\langle V_{\mathrm{p}}^{2}\right\rangle \cdot\langle P(Q)\rangle .
$$

For a system with given particle shape, the polydispersion can be represented by the distribution of the particle volumes. Thus, $\langle P(Q)\rangle$ can be expressed as

$$
\langle P(Q)\rangle=\int \mathrm{d} V(\Delta \rho)^{2} V^{2} P(Q, V) F(V) / \int V^{2} F(V) \mathrm{d} V
$$

where $P(Q, V)$ is the intraparticle structure factor of a particle of volume $V$ and $F(V)$ is the particle size distribution function. For spherical particle $P(Q, V)$ has explicit form of (Eq. (A.4)) with $V=(4 \pi / 3) R^{3}$. Similarly, for cylindrical particles, $P(Q, V)=$ $\int \mathrm{d} \mu|F(\mu, L, R)|^{2}$ with $F(\mu, L, R)$ given as (Eq. (B.7)) and $V=\pi R^{2} L$. The difficulty in analyzing a polydisperse system is often in the choice of a correct distribution function. In this study, we have investigated two specific models : one is the Schultz distribution for spherical particles and the other is the thermal equilibrium distribution for cylindrical aggregates which we just derived in the last section. In both cases, the analytical form of $\langle P(Q)\rangle$ can be derived in a straightforward manner. The results are given in Appendice A and B (see Eqs. (A.5) and (B.6)) for both types of distributions, respectively.

In the following we shall derive $I(Q)$ for these two models :

A. SPHERICAL MODEL. - For spherical particles with Schultz distribution in sizes, the intensity contributed by the particles of radius $R, I(Q, R)$, can be written as

$$
I(Q, R)=N_{\mathrm{p}}(R) \cdot(\Delta \rho)^{2} \cdot\left[(4 \pi / 3) R^{3}\right]^{2} \cdot P(Q, R)
$$

where $P(Q, R)=\left[3 j_{1}(Q R) / Q R\right]^{2}, j_{1}$ is the spherical Bessel function of the first kind. By (Eq. (14)) the total intensity $I(Q)$ can then be obtained by integrating $I(Q, R)$ over $R$ with $N_{\mathrm{p}}(R)$ distributed according to the Schultz distribution function. The explicit form for $I(Q)$ is given in Appendix A (see Eq. (A.5)).

B. CYLINDRICAL MODEL. - For particles formed by aggregation of $N$ molecules of volume $v_{\mathrm{m}}$, the scattering intensity, $I_{N}(Q)$, can be written as, neglecting $S(Q)$,

$$
\begin{aligned}
I_{N}(Q) & =\left(C_{N} / N\right)\left(N v_{\mathrm{m}}\right)^{2}\left[\left(N b_{\mathrm{m}} / N v_{\mathrm{m}}\right)-\rho_{\mathrm{s}}\right]^{2} P_{N}(Q) \\
& =\left(C_{N} / N\right) N^{2}\left(b_{\mathrm{m}}-v_{\mathrm{m}} \rho_{\mathrm{s}}\right)^{2} P_{N}(Q)
\end{aligned}
$$

where $C_{N}$ is the number density of the molecules that form an aggregate containing $N$ molecules, $P_{N}(Q)$ is the intraparticle structure factor of such an aggregate, and $b_{\mathrm{m}}$ is the total scattering length of a molecule. Then, the total intensity $I(Q)$ can be written by summation of $I_{N}(Q)$,

By defining $I(0)$ to be

$$
\begin{aligned}
I(Q) & =\sum_{N=1}^{\infty} \frac{C_{N}}{N} N^{2}\left(b_{\mathrm{m}}-v_{\mathrm{m}} \rho_{\mathrm{s}}\right)^{2} P_{N}(Q) \\
& \equiv I(0) \cdot\langle P(Q)\rangle .
\end{aligned}
$$

$$
\begin{aligned}
I(0) & =\sum_{N=1}^{\infty} N^{2} \frac{C_{N}}{N}\left(b_{\mathrm{m}}-v_{\mathrm{m}} \rho_{\mathrm{s}}\right)^{2} \\
& =\left(b_{\mathrm{m}}-v_{\mathrm{m}} \rho_{\mathrm{s}}\right)^{2} C_{0} \bar{N}_{w},
\end{aligned}
$$


where $C_{0}$ is the total number density of the discotic molecules in the system. Using (Eqs. (5) and (6)) for size distribution $C_{N} /\left(N . C_{0}\right),\langle P(Q)\rangle$ can be derived from (Eq. (16)) to be

$$
\begin{aligned}
\langle P(Q)\rangle & =\sum_{N=1}^{\infty} N C_{N}\left(b_{\mathrm{m}}-v_{\mathrm{m}} \rho_{\mathrm{s}}\right)^{2} P_{N}(Q) / \sum_{N=1}^{\infty} N C_{N}\left(b_{\mathrm{m}}-v_{\mathrm{m}} \rho_{\mathrm{s}}\right)^{2} \\
& =\sum_{N=1}^{\infty} N^{2} X_{N} P_{N}(Q) / \sum_{N=1}^{\infty} N^{2} X_{N} \\
& =\sum_{N=1}^{\infty} \beta^{N} N^{2} P_{N}(Q) / \sum_{N=1}^{\infty} \beta^{N} N^{2} .
\end{aligned}
$$

Equations (16)-(18) are the equations to be used for analysis of SANS data. The explicit form of $P_{N}(Q)$ in (Eq. (18)) for an aggregate containing $N$ stacked HET- $n$ molecules (the scattering geometry is a cylindrical shell (see Fig. 1)) is given in Appendix B (Eq. (B.6)).

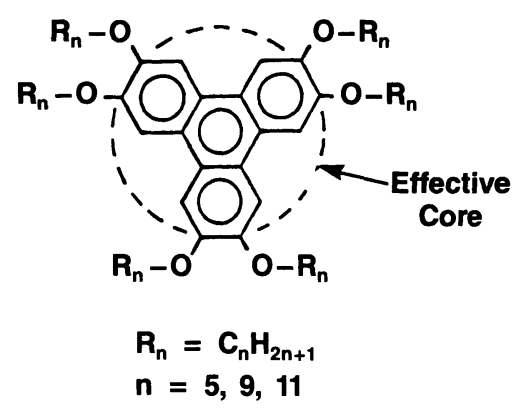

Fig. 1a. - HET- $n$ molecular structure. The length of the aliphatic side chains can be varied chemically. Three different chain lengths with $n=5,9$, and 11 were synthesized for this study.

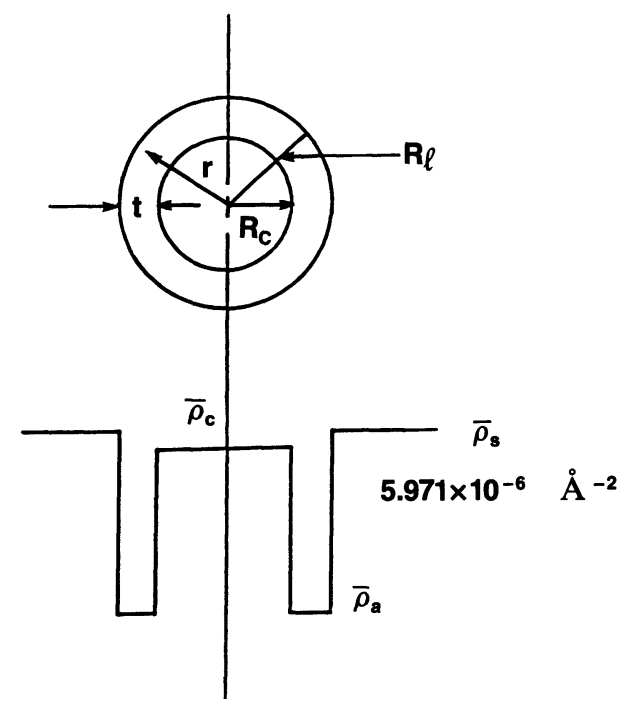

Fig. 1b. - Structural model for stacked HET- $n$ aggregates of length $L$. The concentric circle is the top view of the cylindrical aggregate which includes two regions : the aromatic core region and the aliphatic chain layer. The corresponding scattering length profile show below indicates that the main contrast comes from the aliphatic chain layer and solvent and the essential scattering geometry is a cylindrical shell. 


\section{Experimental data and analysis.}

The SANS measurements of the three sets of HET- $n$ solutions (Tab. I) all showed strong small angle scattering intensities. Typical results are shown in figure 2 for HET-5 in the Guinier plots (i.e., $\ln I(Q)$ vs $Q^{2}$ ). These plots show no linear region, indicating that either the particles are not monodisperse spheres or the $Q$ range is beyond the Guinier region. Thus, a more sophisticate structural analysis is needed. We also note that, by increasing HET-5 concentration, the scattering intensity first increases (see curves 1 and 2 of Fig. 2), then drops (curves 3 and 4), and finally increases steadily again (curves 4, 5 and 6). This behavior, as shown in the inset of figure 2 for the scattering intensity at $Q=0.03 \AA^{-1}$ for HET-5, was similarly observed for HET-9 and HET-11 with the transitions taking place in the solute
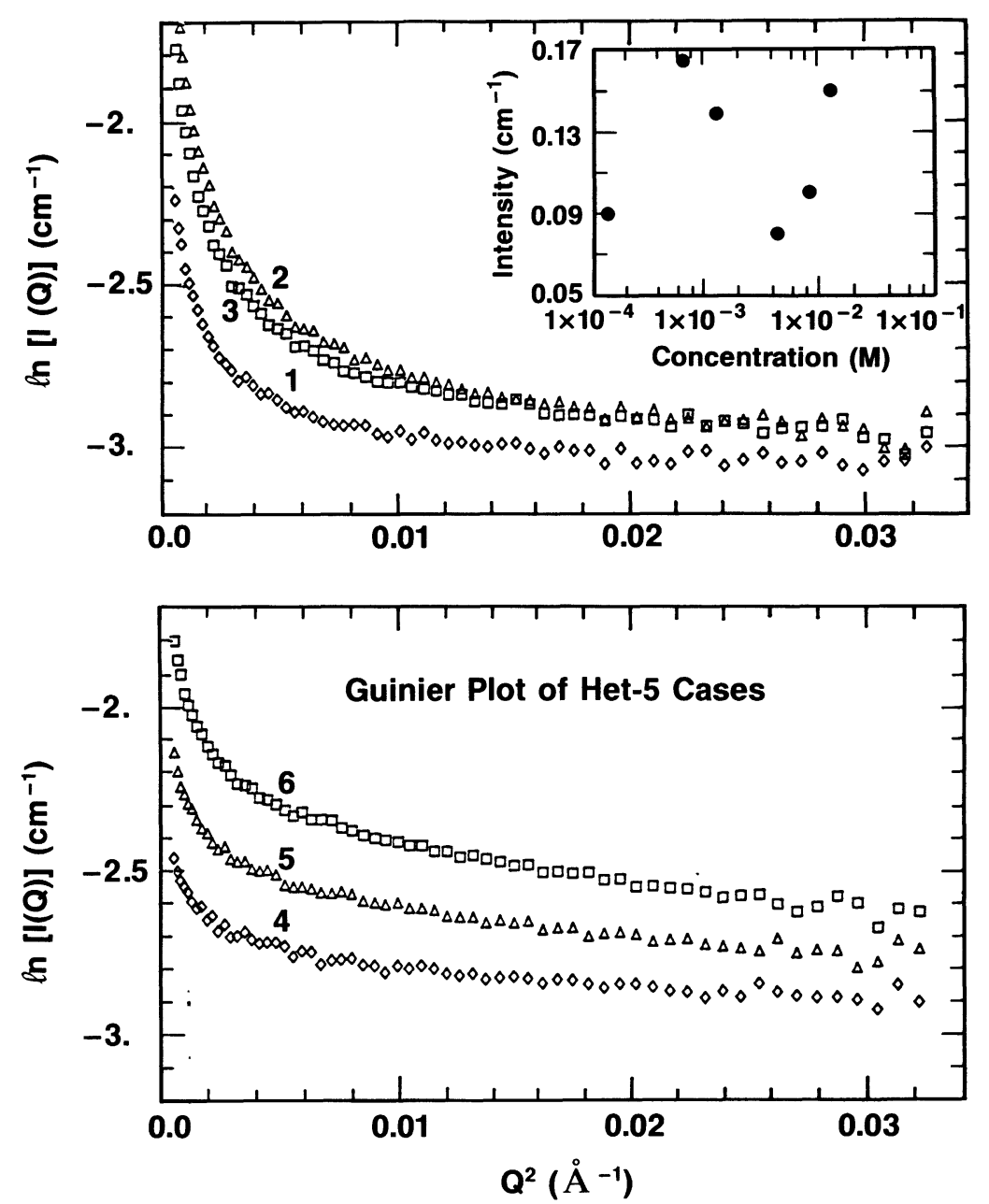

Fig. 2. - Guinier plot of HET-5 in hexadecane at various concentrations : (1) $1.3 \times 10^{-4} \mathrm{M}$, (2) $6.5 \times 10^{-4} \mathrm{M}$, (3) $1.3 \times 10^{-3} \mathrm{M}$, (4) $4.3 \times 10^{-3} \mathrm{M}$, (5) $8.6 \times 10^{-3} \mathrm{M}$, and (6) $1.3 \times 10^{-2} \mathrm{M}$. At low concentration the scattering intensity increases first and then drop (upper panel). As concentration further increases the intensity scales as concentration (lower panel). The intensities at $Q=0.03 \AA^{-1}$ for as a function of concentration is given in inset. 
concentrations range of $1.3 \times 10^{-3} \mathrm{M}$ to $4.3 \times 10^{-3} \mathrm{M}$ for HET-5 $\left(9.26 \times 10^{-4} \mathrm{M}\right.$ to $3.06 \times 10^{-3} \mathrm{M}$ for HET-9 and $8.4 \times 10^{-4} \mathrm{M}$ to $2.64 \times 10^{-3} \mathrm{M}$ for HET-11). We thus define the concentration below this transition concentration to be low concentration range and that above to be high concentration range. We attribute this behavior to some type of structural transition to be discussed later.

We now discuss the results of our data analysis in two parts : the spherical model and the columnar shell model.

A. SPHERICAL MODEL. - To investigate the possible structural model for the aggregates, we first analyzed the SANS data assuming the aggregates to be spherical with the size distribution according to a Schultz distribution. This distribution has been widely applied in polymer solutions $[20,21]$. The analysis was carried out by using the formalism outlined in section 4.A and Appendix A with three adjustable parameters, namely, average particle radius $\bar{R}$, the width parameter $z$ of the distribution, and an amplitude factor. The amplitude factor was introduced because the scattering length density of a spherical aggregate formed by discotic molecules can not be calculated a priori as in the stacked cylinder case (see section below). Due to this additional parameter the fittings may not be unique and multiple convergence may occur. In order to avoid multiple convergence we systematically changed $\bar{R}$ and used only $z$ and the amplitude factor as free parameters to fit our SANS data. Typical results of the curve fitting are shown in figures $3 a$ and $3 b$ for the case of HET-5 at low and high concentrations respectively. The parameters $(\bar{R}$ and $z)$ extracted from Schultz model fittings for all three sets of samples are given in table $I$.

For comparison, the results of curve fitting using a monodisperse sphere model is also shown in figure 3. One sees that the monodisperse sphere model is not acceptable. The quality of the fitting is greatly improved when the Schultz distribution is incorporated. However, the values of the width parameter, $z$, so obtained are relatively low (in the range of -0.5 to 3 , see Tab. I). The equivalent width of the radius distribution is about 2.8 to 1.0 times of the mean radius. That is to say the system would be extremely polydisperse

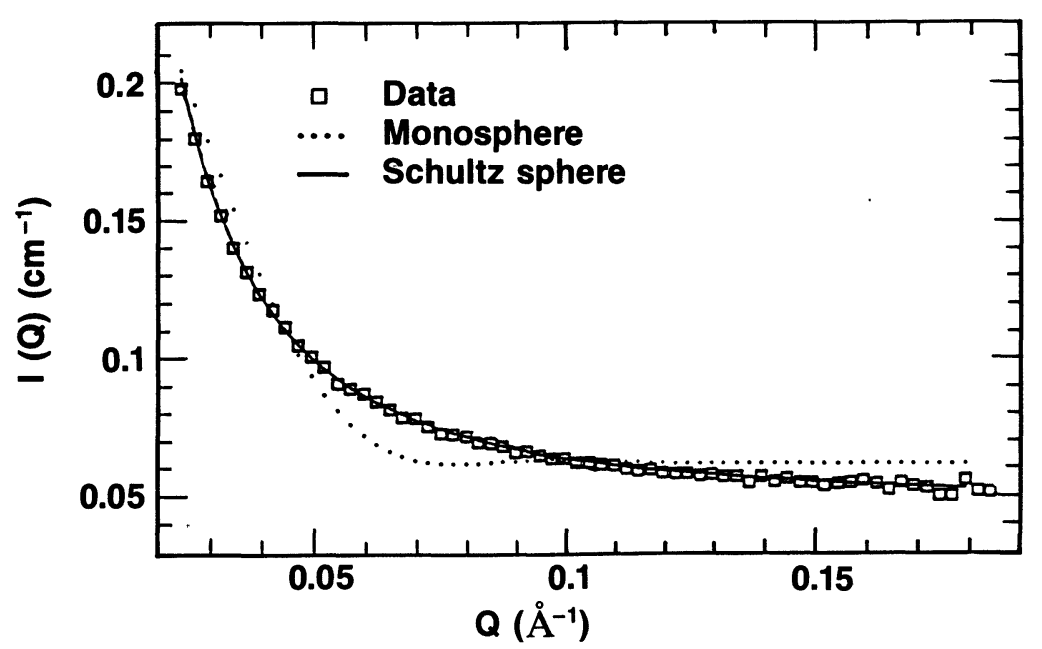

Fig. 3a. - Model fitting of HET-5 in hexadecane at $6.5 \times 10^{-4} \mathrm{M}$ using various structural models. The Schultz gives the best fit. However, the intensities calculated by using the extracted parameters and the molecule-solvent contrast are substantially higher ( $>3$ times) than the measured scattering intensities. We thus consider this model to be only qualitatively meaningful. 


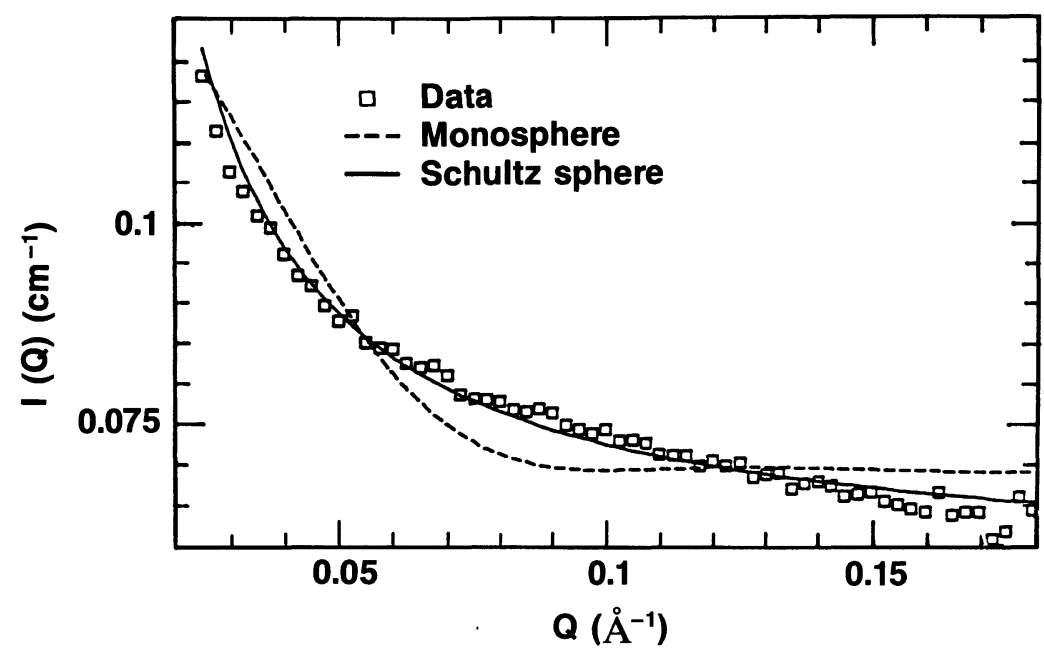

Fig. 3b. - Same fitting as 3a for $8.6 \times 10^{-3} \mathrm{M}$. The Schultz fitting does not show as good fit as it does for the low concentration case shown in 3a. Again, the calculated intensities are too much higher than the measured absolute intensities. This suggests that the polydisperse spherical model may not be applicable for high concentration cases.

( $p=1 / \sqrt{1+z}$, see Eq. (A.3)) based on this model. For such high polydispersion the true meaning of spherical particles can no longer be assumed. It could equally be possible that the system contains particles with very irregular shapes, particularly, in the low concentration cases. To our experiences, spherical particles with Schultz distribution is a relatively flexible model and is able to accommodate the irregularity of the particle shape by decreasing the width parameter (or increase the polydispersity). One solution to this dilemma is to compare the absolute scattering intensity. To do this we took the particle-solvent contrast as that between the discotic molecule (average over core aromatic core and the aliphatic chains) and the deuterated hexadecane. Using the structural parameter values extracted from spherical Schultz model the absolute scattering intensity were then calculated. The intensities thus calculated is much higher ( $>3$ times) at all $Q$ than that obtained from measurements. We therefore believe that the fitting using spherical Schultz model may only be qualitatively meaningful. We will come back to discuss this point after showing the results of the columnar shell model.

B. Columnar Shell MOdel. - Before we performed the model fitting we plotted the Porod plot for the thin rod particle, $\ln [Q . I(Q)]$ vs $Q^{2}$ (see Fig. 4), for the case of HET-5 to examine the large $Q$ behavior. If the particles are monodisperse cylinder with large aspect ratio the curve should show linear behavior at large $Q$ region. However, no linear behavior is shown in figure 4 at any $Q$ region, which readily indicates that the particles are not monodisperse cylinders of high aspect ratio. Actually, the nonlinearity of figure 4 comes from the fact that most of the particles are of low aspect ratios. We will come back to discuss this point after structural analysis using columnar shell model.

We now consider curve fitting using columnar shell model for the aggregates (see Fig. 1). Using a CPK precision molecular model [22], the neutron scattering length density, and thus particle-solvent contrast, of such an aggregate can be calculated (see Appendix B). Therefore, the absolute scattering intensity in this model can be precisely computed. Using this model for data analysis, we first calculated $P_{N}(Q)$ by use of (Eqs. (B.6)-(B.8)) and then 

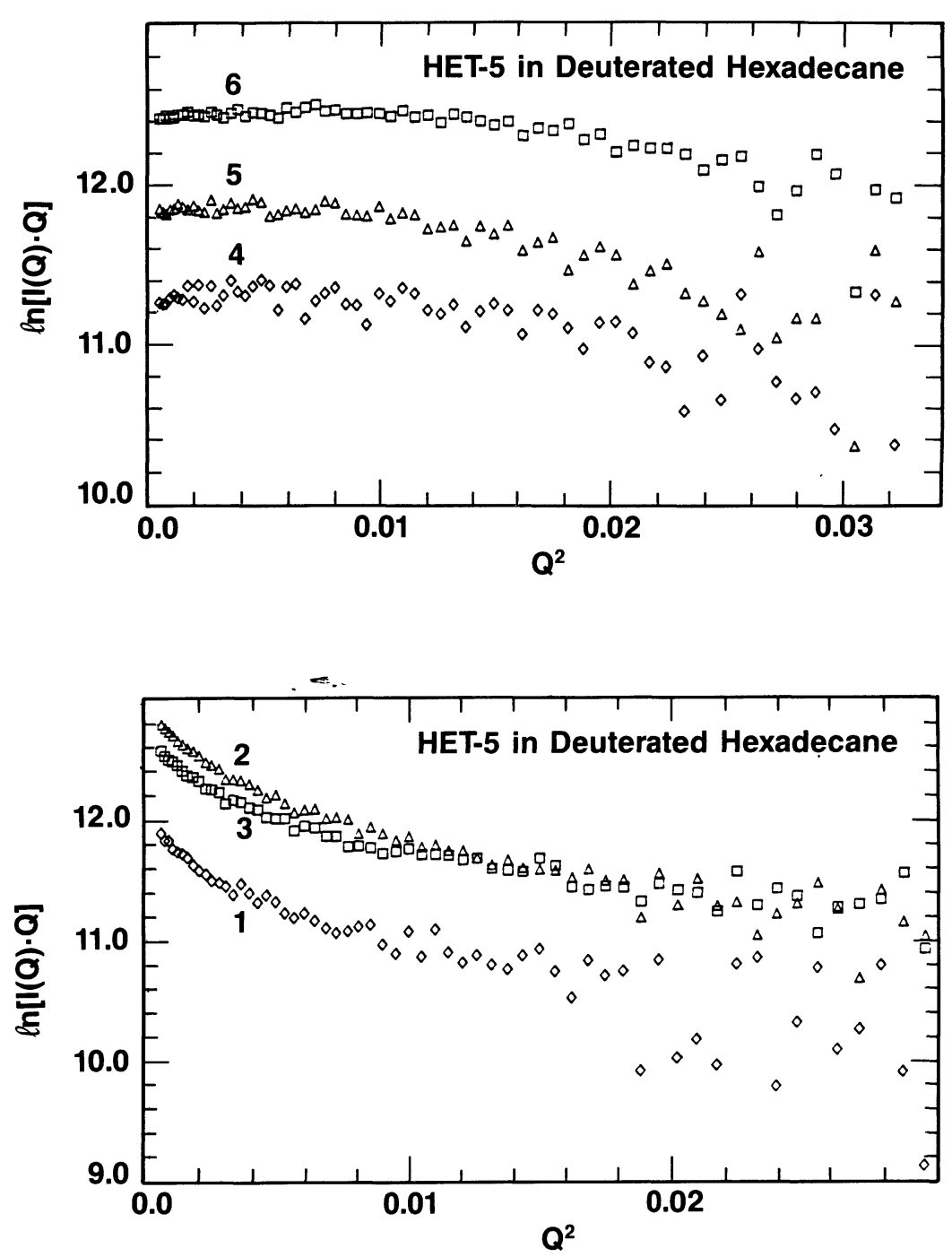

Fig. 4. $-\ln [Q . I(Q)] v s . Q^{2}$ plot. This plot would show linear behavior at large $Q$ region if the particles are thin rods and are monodisperse in size. It is obvious that no distinctive linear behavior exhibits at large $Q$ for all the spectra (1-6). This is because most of the particles are not thin-rod like and the particle sizes are polydisperse (see Sect. 5).

computed the scattering intensity $I(Q)$ according to (Eqs. (15) and (16)). The calculated scattering intensity was then fitted to the SANS data using $\beta$ (Eq. (6)), $d$ (Eq. (B.2)), and $R_{\ell}$ (see Fig. 1 ) as adjustable parameters. Again, we systematically changed $R_{\ell}$ and used only $\beta$ and $d$ as adjustable parameters in the fitting so that multiple convergence can be avoided. From the extracted $\beta$ and $d$ values we calculated the basic thermodynamic parameter $\delta$ (the formation energy) by (Eq. (8)) and the structural parameter $\bar{L}$ (the average particle length) by (Eq. (B.3)). The results of data fitting for HET-5, HET-9, and HET-11 in the high concentration range are shown in (Figs. 5 to 7), respectively. The values of the extracted and the subsequently calculated parameters are tabulated in table I. 


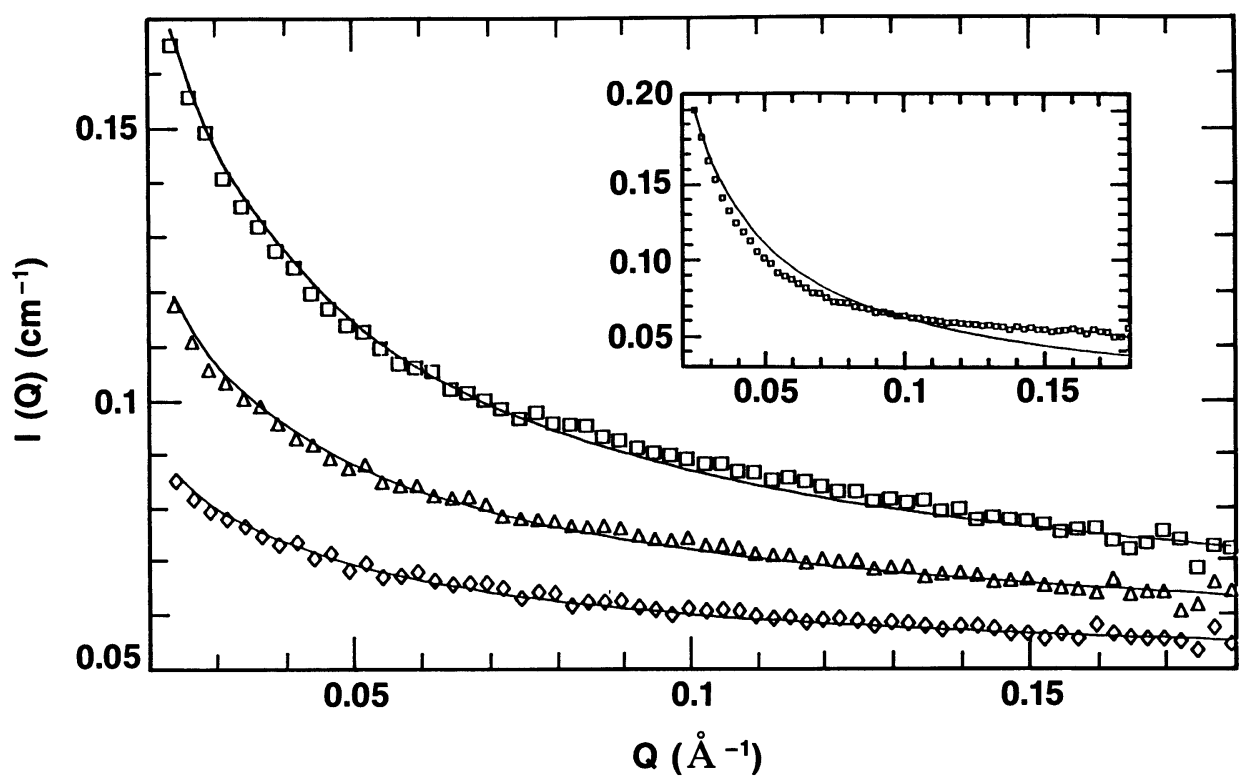

Fig. 5. - SANS data fitting of HET-5 in deuterated hexadecane of various concentrations : ( $\square$ ) $1.3 \times 10^{-2} \mathrm{M},(\triangle) 8.6 \times 10^{-3} \mathrm{M}$, and $(\diamond) 4.3 \times 10^{-3} \mathrm{M}$ using rod-like model with particle size distribution according to that derived in section 3 . The qualities of fits are reasonable but not excellent because (1) the molecular stackings are hardly perfect, (2) an average scattering length density, $\bar{\rho}_{\mathrm{a}}$, was used for aliphatic chain region, and (3) the aggregates are expected to have some fluctuation in shape.

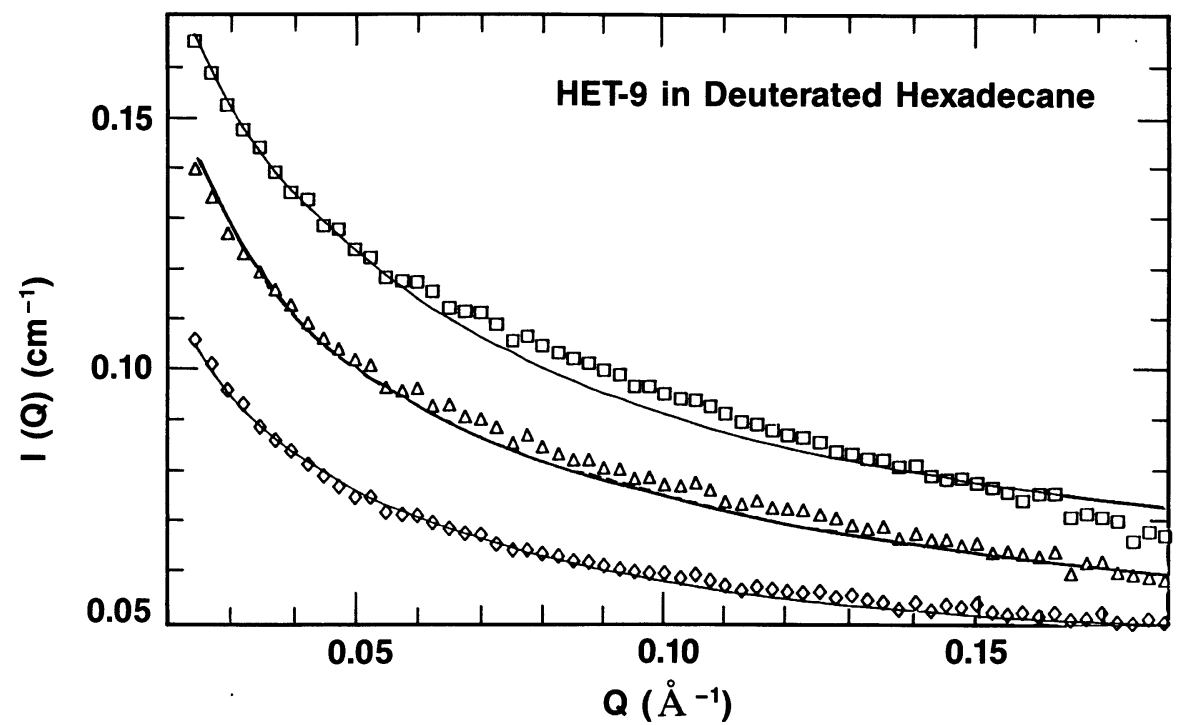

Fig. 6. - Same as figure 6 for HET-9 at (口) $9.26 \times 10^{-3} \mathrm{M},(\triangle) 6.1 \times 10^{-3} \mathrm{M}$, and $(\diamond)$ $3.06 \times 10^{-3} \mathrm{M}$. The qualities of the fits are attributed to the same reasonings as given in figure 6 . 


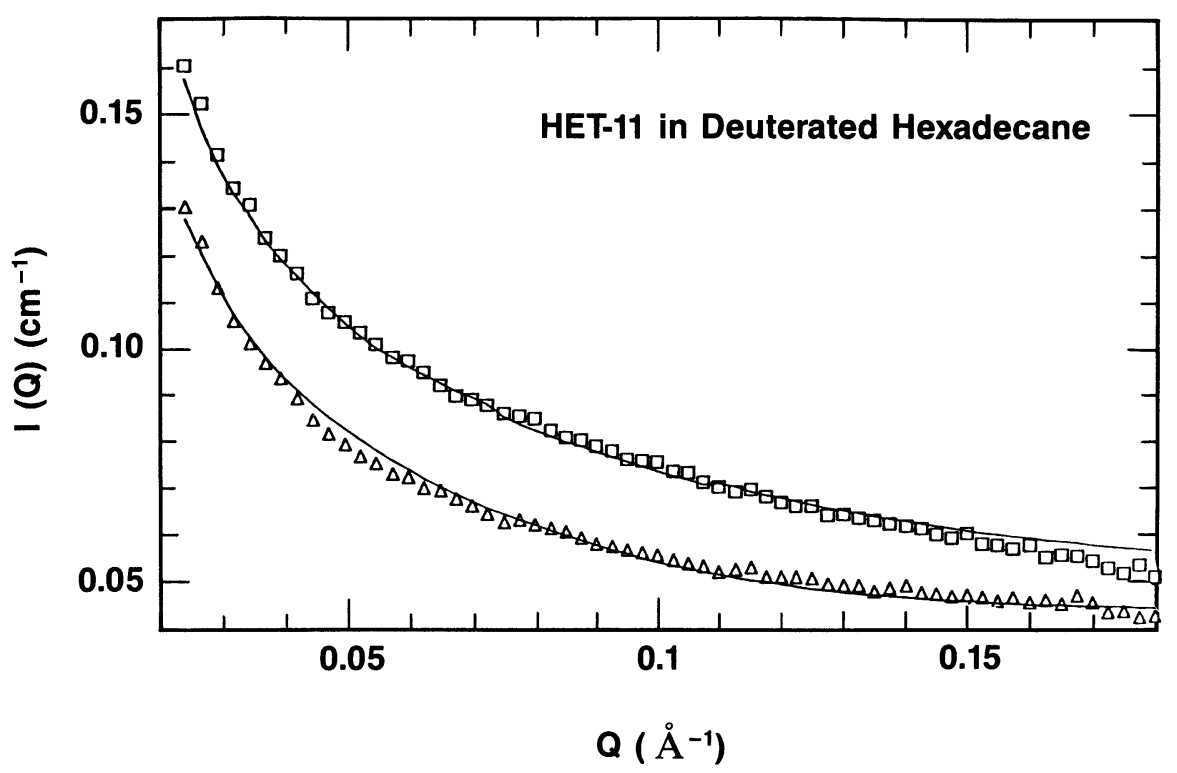

Fig. 7. - Same as figure 6 for HET-11 at (口) $5.28 \times 10^{-3} \mathrm{M}$ and $(\triangle) 2.64 \times 10^{-3} \mathrm{M}$.

Based on these data fittings we found that all the high concentration cases in three systems can be reasonably fitted by the columnar shell model at absolute intensity scale. However, the fits are quite poor for low concentration region, which show abnormal intensity-concentration dependence (Fig. 2). A typical example is shown in the inset of figure 5 .

C. THE PHYSICAL MODEL OF Discotic AGgREgATION. - By now, we have presented the results of curve fitting based on two different models in both low and high concentration regions. The most promising results are shown in the columnar shell model for the high concentrations. Combining these results with those from spherical model fittings, we might argue that the molecules start to aggregate at low concentration in a very irregular manner, which results in the discrepancy of absolute intensity as we found in section V.A, because the molecular packing are much loose than compact packing. As concentration increases the discotic molecules start to stack regularly and the aggregates become rodlike. This amount to saying that there is a transition of particle geometry such as that commonly observed in micellar solution (sphere to rod transition) [23].

We consider a qualitative argument that might be applied to explain this transition. The association energies of HET- $n$ molecules are mainly derived from dipole-dipole and quadrupole-quadrupole interactions. Thus, the resultant structure of the aggregate would solely depend upon the competition between these two forces. Our SANS data suggest that the quadrupole interactions predominate at low concentration to lead the aggregate structure to irregular shapes via both vertical and horizontal associations. In this way, the rotational entropy and the chain-solvent affinity dominate the free energy of the system. As concentration increases the dipole-dipole attractions between aromatic cores become predominent and lead the discotic molecules to stack. The aggregates thus form into cylindrical shape.

Based on the analysis using cylindrical shell model we found that all the values of the extracted parameters are physically meaningful. In the following we shall discuss these 
parameters. The $\beta$ values extracted are very close to unity indicating that the particles are polydisperse in sizes. From this fact and that more small particles (low aspect ratios) than the large particles in the solution at any concentration $\left(X_{N}\right.$ is a monotonic decaying function for a given $\beta<1$, see Eq. (6)) one can explain why figure 4 fails to show linear behavior at large $Q$ region. This is because Porod plot requires large aspect ratio for all particles in the system. The extracted dimer formation energy, $\delta$, was found to be nearly constant ( $\sim-13 K_{\mathrm{B}} T$ or $-7.6 \mathrm{kcal}$ ) within each discotic system which is consistent with the ladder model. The slight changes of $\delta$ values as a function of concentrations (from - 13.01 to $-13.06 K_{\mathrm{B}} T$ for HET-5, -12.61 to $-12.40 K_{\mathrm{B}} T$ for HET-9 and -12.26 to $-12.43 K_{\mathrm{B}} T$ for HET-11) is attributed to the changes of intermolecular distance $d$ 's (see Tab. I). One also notes that $\delta$ value decreases with aliphatic chain length from HET-5 to HET-11, which is expected because the longer the aliphatic chain the stronger its affinity to the solvent. Similarly, the core-to-core distance, $d$, increases with aliphatic chain length and the values ( $\sim 5.6$ to $6.3 \AA$ ) are much greater than that in pure discotic liquid crystal phase (3.6 $\AA$ ) [6, 7]. The $\bar{N}_{\text {w }}$ and $\bar{N}_{N}$ so obtained increase as concentration increases indicating the growth of the aggregates (see Tab. I). The cross sectional radius of the stacked cylinder, $R_{\ell}$, slightly decreases with discotic concentration, which may be attributed to the increasing regularity of the molecular stackings. Finally, we approximately estimate the dimer formation energy by calculating the London forces (neglecting the quadrupole forces and solvent effect) between two HET- $n$ molecules (assuming the molecule to be a plate) [24]. Taking Hamaker constant of benzene for HET- $n$ molecule and inter-plate distance to be $6 \AA$, the London force is at order of $15 K_{\mathrm{B}} T$. With this rough estimation we believe that the formation energy extracted $\left(\sim 13 K_{\mathrm{B}} T\right)$ is quite reasonable.

Recently there was a luminescence experiment carried out by Markovitsi et al. on HET-6 in dichrolomethane solution at the concentration range similar to our cases [29]. In this experiment no self-associations were observed unlike the results found in this study. This is because the solvents used are very different. Their results, together with our results, provides a good indication that the self-association is a energy driven process and its occurrence depends on the energy competition between the intermolecular interactions and the molecule-solvent interactions.

\section{Conclusions.}

We have demonstrated that the discotic molecules HET-5, HET-9, and HET-11 have strong propensity to self-associate in deuterated hexadecane. The aggregates are irregular in shape at low concentration and become rod-like at higher concentration. The columnar aggregates are formed by stackings of the disc-like molecules with inter-core distance $\sim 6 \AA$. The size distribution of these aggregates is describable by an equilibrium thermodynamic model derived from the first principle. The dimer formation energies, taking into account the effect of molecule-solvent affinity, are extracted to be about $13 K_{\mathrm{B}} T$ (or $7.6 \mathrm{kcal}$ ) and only slightly concentration dependent which is consistent with the value estimated on the base of the London force interaction.

\section{Acknowledgement.}

The authors wish to thank Drs. S. K. Sinha and C. R. Safinya for useful discussions. One of us (EYS) would like to thank Dr. D. K. Schneider and B. P. Schoenborn for help in the SANS measurements at the Department of Biology in Brookhaven National Laboratory. 


\section{Appendix A : Spherical particle with the Schultz distribution.}

Schultz distribution has been used extensively in describing the molecular weight distribution of polymer systems $[20,21]$. It is a two parameter distribution,

$$
F_{\mathrm{s}}(R)=\left[\frac{z+1}{\bar{R}}\right]^{z+1} R^{z} \exp \left[-\left[\frac{z+1}{\bar{R}}\right] R\right] \frac{1}{\Gamma(z+1)}
$$

where $\bar{R}$ is the average radius of the spherical particles, $z>-1$ [25] is the width parameter characterizing the degree of polydispersion of the particle sizes, and $\Gamma(x)$ is the gamma function. Schultz distribution is skewed to the larger sizes, tending to a Gaussian form at large $z$ value and approaches to a delta function as $z$ approaches infinity. Since $j$-th moment of the Schultz distribution can be easily calculated by

$$
\left\langle R^{j}\right\rangle=\frac{\Gamma(z+j+1)}{\Gamma(z+1)}\left[\frac{\bar{R}}{z+1}\right]^{j}
$$

the polydispersity index $p$ can be defined and derived to be

$$
p \equiv \sqrt{R^{2}-\bar{R}^{2}} / \bar{R}=1 / \sqrt{z+1} .
$$

The weighted intra-particle structure factor for spherical particles with Schultz distribution in sizes can be expressed as [25]

$$
P(Q)=\int_{0}^{\infty} \mathrm{d} R F_{\mathrm{s}}(R)\left[\frac{3 j_{1}(Q R)}{Q R}\right]^{2}
$$

This integration can be carried out analytically as [26],

$$
P(Q)=8 \pi^{2}(\Delta \rho)^{2} \bar{R}^{6}(z+1)^{-6} \alpha^{z+7} \mathfrak{J}(Q)
$$

where

$$
\begin{aligned}
J(Q)= & \alpha^{-(z+1)}-\left(4+\alpha^{2}\right)^{-(z+1) / 2} \cos \left(\xi_{1}\right)+(z+2)(z+1)\left[\alpha^{-(z+3)}+\right. \\
& \left.+\left(4+\alpha^{2}\right)^{-(z+3) / 2} \cos \left(\xi^{3}\right)\right]-2(z+1)\left(4+\alpha^{2}\right)^{-(z+2) / 2} \sin \left(\xi_{2}\right) \\
\xi_{i}= & (z+i) \cdot \tan ^{-1}(2 / \alpha) \\
\alpha= & (z+1) / Q \bar{R} .
\end{aligned}
$$

\section{Appendix B : Particle form factor of stacked disk-like molecules.}

To compute the averaged particle form factor of an aggregate formed by stacking HET- $n$ molecules in thermal equilibrium we assume that this aggregate can be approximated by an effective cylinder containing two regions (see Fig. 1). Then, the distribution of the aggregate sizes should, in principle, follow that derived previously (Eqs. (6) and (7)). If the stackings are regular then there are clearly two regions in a stacked cylinder, namely, the central core 
region of radius $R_{\mathrm{c}}$ and the aliphatic chain layer of thickness $t$. Using a CPK precision molecular model [22] the core radius and its volume can be calculated to be $4.4 \AA$ and $184.9 \AA^{3}$ respectively. The fully stretched chain length was calculated to be $8 \AA$ for HET-5, $14 \AA$ for HET-9, and $16.6 \AA$ for HET-11. The corresponding volumes (volume of six aliphatic chains in a molecule) are respectively $933 \AA^{3}, 1680 \AA^{3}$, and $2053 \AA^{3}$. Figure 1a depicts this structural model and figure $1 \mathrm{~b}$ shows the corresponding scattering length density profile. It is clear from the scattering density profile that the coherent scattering is largely contributed by the aliphatic chains and the scattering geometry is essentially a cylindrical shell.

To calculate $P_{N}(Q)$ of such a cylindrical shell stacked by $N$ molecules, the average scattering length density of the core, $\bar{\rho}_{c}$, and that of the aliphatic chain region, $\bar{\rho}_{\mathrm{a}}$, have to be formulated. Since $\bar{\rho}_{\mathrm{c}}$ depends on the core-to-core distance, which has been reported to be $3.6 \AA$ in liquid crystal phase $[6,7,27]$ (this core-to-core distance may not be retained in isotropic solution phase) we write

$$
\bar{\rho}_{\mathrm{c}}=\frac{N \sum_{\mathrm{m}} b_{\mathrm{m}}^{\mathrm{c}}}{d(N-1) \pi R_{\mathrm{c}}^{2}}=\frac{N \sum_{\mathrm{m}} b_{\mathrm{m}}^{\mathrm{c}}}{L \pi R_{\mathrm{c}}^{2}}
$$

where $\sum_{\mathrm{m}} b_{\mathrm{m}}^{\mathrm{c}}$ is the total scattering length of the core, $d$ is the core-to-core distance, and $R_{\mathrm{c}}$ is the core radius. $L$ is the length of the cylinder relating to $d$ and $N$ according to

$$
L=d(N-1)+3.04 \quad(\AA),
$$

or in the case of polydisperse systems,

$$
\dot{\bar{L}}=d\left(\bar{N}_{\mathrm{w}}-1\right)+3.04 \quad(\AA)
$$

where $3.04 \AA$ is the thickness of the aromatic core obtained from measuring CPK precision molecular model [22]. In aliphatic chain region, the scattering length density is a function of, besides $d$, the thickness of the aliphatic chain region, $t$, (it is not necessary to be the stretched length of the aliphatic chain for molecules in solution), and $r\left(R_{\mathrm{c}}<r<R_{\ell}\right.$, see Fig. 1a). Since the stacking is expected to be not perfect and that there is alkylchain flexibility, which was even observed in discotic columnar mesophases [28], we thus eliminated the $r$ dependence by taking an averaged scattering length density for this region,

$$
\bar{\rho}_{\mathrm{a}}=\frac{N \sum_{\mathrm{m}} b_{\mathrm{m}}^{\ell}}{V_{\mathrm{sh}}}
$$

where $\sum_{\mathrm{m}} b_{\mathrm{m}}^{\ell}$ is the total scattering lengths of the six aliphatic chains attached to the core and

$$
V_{\mathrm{sh}}=(N-1) d \pi\left(R_{\ell}^{2}-R_{\mathrm{c}}^{2}\right)=L \pi\left(R_{0}^{2}-R_{\mathrm{c}}^{2}\right)
$$

is the shell volume. In equation (B.5) $R_{\ell}$ represents the apparent radius of the cylinder (see Fig. 1b) to be determined through data fitting.

With equation (B.3)-(B.5) $P_{N}(Q)$ of such a cylindrical shell can be written as

$$
P_{N}(Q)=\frac{1}{2} \int_{-1}^{1} \mathrm{~d} \mu\left[f F\left(\mu, L, R_{\mathrm{c}}\right)+(1-f) F\left(\mu, L, R_{\ell}\right)\right]^{2} .
$$


where $\mu=\cos \theta$ ( $\theta$ is the scattering angle) and $F(\mu, L, R)$ is the particle form factor of a cylindrical aggregate,

$$
F(\mu, L, R)=\frac{\sin (Q \mu L / 2)}{Q \mu L / 2} \frac{2 J_{1}\left(Q R \sqrt{1-\mu^{2}}\right)}{Q R \sqrt{1-\mu^{2}}},
$$

and

$$
f=\Delta \bar{\rho}_{\mathrm{c} \ell} /\left(\Delta \bar{\rho}_{\mathrm{cl}}+\Delta \bar{\rho}_{\ell \mathrm{s}}\right)
$$

is the normalization factor, $\Delta \bar{\rho}_{\mathrm{c} \ell}=\bar{\rho}_{\mathrm{c}}-\bar{\rho}_{\ell}$ is the scattering contrast between core region and aliphatic chain region and $\Delta \bar{\rho}_{\ell_{\mathrm{S}}}=\bar{\rho}_{\ell}-\bar{\rho}_{\mathrm{s}}$ is that between aliphatic chain region and solvent.

\section{References}

[1] For a review see Lindman B. and Wennerst H., T. Curr. Chem., 87 (1980) 1-83.

[2] Nir S., Bentz J., Wilschut J. and Duzgunes N., Prog. Surf. Sci. 13 (1983) 1-124.

[3] For a review see several articles in Physics of Complex and Supermolecular Fluids, ed. by S. A. Safran and N. A. Clark Wiley Inter-Science (1987).

[4] Chandrasekhar S., Shadashiva B. K., Suresh K. A., Pramana 9 (1977) 471.

[5] For a review, see BILlARD J., in Liquid Crystals of One-end Two-Dimensional Order, edited by H. Helfrich and G. Heppke (Springer-Verlag, 1980) pp. 383.

[6] Levelut A. M., in Proceedings of the International Liquid Crystal Conference, Bangalore, 1979 (Wiley, New York, 1980).

[7] Safinya C. R., Liang K. S., Varady W. A., Clark N. A. and Anderson G., Phys. Rev. Lett. 53 (1984) 1172.

[8] Saeva F. D. and Reynolds G. A., Mol. Cryst. Liq. Cryst. 132 (1986) 29.

[9] Gasparoux (H.), in reference [2], pp. 373.

[10] Brooks J. D. and TAYlor G. H., Carbon 3 (1965) 185.

[11] Chiang L. Y., Safinya, C. R., Clark N. A., Liang K. S. and Block A. N., Chem. Comm. (1985) 694.

[12] Tinh N. H., Dubois J. C., Malthete J. and Destrade C., C. R. Hebd. Acad. Sci., Paris 286 C (1978) 463.

[13] Chen S. H., Lin T. L., « Application of Small Angle Neutron Scattering to Problems in Colloidal Solutions » Ch. 16 in Thermal Neutron Scattering Vol. II, ed. by D. L. Price and K. Skold, Academic Press N.Y. (1987).

[14] TANFORD C., Hydrophobic Effects, Wiley, New York (1980).

[15] Ben-Shaul A. and Gelbart W. M., J. Phys. Chem. 86 (1986) 316.

[16] Tausk R. J. M., Karmiggelt J., Oudshoorn C., Overbeek J. Th. G., Biophys. Chem. 1 (1974) 175.

[17] Porod G., in Small Angle X-ray Scattering, pp. 17, Ed. by O. Glatter and O. Kratky, Academic Press New York (1982).

[18] See several review articles in Physics of Amphiphiles: Micelles, Vesicles and Microemulsions, ed. by V. Degiorgio and M. Corti, North-Holland, New York (1985).

[19] HAYTer J. B., « SANS Studies of Micellar and Magnetic Fluids » pp. 21 in Physics of Complex and Supermolecular Fluids ed. by S. A. Safran and N. A. Clark, John Wiley \& Son, N.Y. (1987).

[20] Zimm B. H., J. Chem. Phys. 16 (1948) 1099.

[21] Ford N. C. Jr., Gabler R. and Karasz F. E., in Polymer Molecular Weight Methods ed. by R. E. Could, ADCSAJ 125 (1973).

[22] The details of this precision molecular model are given in the company booklet «CPK Precision Molecular Models » of The Ealing Co., 22 Pleasant street, South Natick, MA. 01760 U.S.A.

[23] Israelachvili J. N., Mitchell D. J., Ninham B. W., J. Chem. Soc. Faraday Trans. II, 72 (1976) 1525. 
[24] Hiemenz P. C., Principles of Colloid and Surface Chemistry, 2nd ed., Marcel Dekker Inc., NY (1986).

[25] MAGid L., Colloid and Surfactant, 19 (1986) 129.

[26] Kotlarchyk M., Chen S. H., Huang J. S. and Kim M. W., Phys. Rev. A 29 (1984) 2054.

[27] Levelut A. M., J. Phys. France 40 (1979) L81.

[28] Destrade C., Nguyen Hunn Tinh, Malthete J. and Levelut A. M., J. Phys. France. 44 (1983) 597.

[29] Markovitsi D., Rigaut F., Mouallem M. and Malthete J., Chem. Phys. Lett. 135 (1987) 236. 